\title{
Improving tuberculosis surveillance by detecting international transmission using publicly available whole genome sequencing data
}

\author{
Andrea Sanchini ${ }^{1,2}$, Christine Jandrasits ${ }^{2,3}$, Julius Tembrockhaus ${ }^{3}$, Thomas Andreas Koh $\mathbf{4}^{4,5}$, Christian Utpatel ${ }^{4,5}$, Florian P \\ Maurer $^{6}$, Stefan Niemann ${ }^{4,5}$, Walter Haas ${ }^{1}$, Bernhard Y Renard ${ }^{3,7}$, Stefan Kröger ${ }^{1,8}$ \\ 1. Respiratory Infections Unit (FG36), Department of Infectious Disease Epidemiology, Robert Koch Institute, Berlin, Germany \\ 2. These authors contributed equally to this manuscript \\ 3. Bioinformatics Unit (MF1), Department of Methodology and Research Infrastructure, Robert Koch Institute, Berlin, Germany \\ 4. Molecular and Experimental Mycobacteriology, Research Center Borstel, Borstel, Germany \\ 5. German Center for Infection Research (DZIF), partner site Hamburg - Lübeck - Borstel - Riems, Germany \\ 6. National and WHO Supranational Reference Laboratory for Mycobacteria, Research Center Borstel, Borstel, Germany \\ 7. Hasso Plattner Institute, Faculty for Digital Engineering, University of Potsdam, Potsdam, Germany \\ 8. German Center for Infection Research (DZIF), partner site Hannover - Brunswick, Germany
}

Correspondence: Bernhard Y Renard (Bernhard.renard@hpi.de)

Citation style for this article:

Sanchini Andrea, Jandrasits Christine, Tembrockhaus Julius, Kohl Thomas Andreas, Utpatel Christian, Maurer Florian P, Niemann Stefan, Haas Walter, Renard Bernhard Y, Kröger Stefan. Improving tuberculosis surveillance by detecting international transmission using publicly available whole genome sequencing data. Euro Surveill. 2021;26(2):pii=1900677. https://doi.org/10.2807/1560-7917.ES.2021.26.2.1900677

Introduction: Improving the surveillance of tuberculosis (TB) is especially important for multidrug-resistant (MDR) and extensively drug-resistant (XDR) TB. The large amount of publicly available whole genome sequencing (WGS) data for TB gives us the chance to re-use data and to perform additional analyses at a large scale. Aim: We assessed the usefulness of raw WGS data of global MDR/XDR Mycobacterium tuberculosis isolates available from public repositories to improve TB surveillance. Methods: We extracted raw WGS data and the related metadata of M. tuberculosis isolates available from the Sequence Read Archive. We compared this public dataset with WGS data and metadata of 131 MDR- and XDR M. tuberculosis isolates from Germany in 2012 and 2013. Results: We aggregated a dataset that included 1,081 MDR and 250 XDR isolates among which we identified 133 molecular clusters. In 16 clusters, the isolates were from at least two different countries. For example, Cluster 2 included 56 MDR/XDR isolates from Moldova, Georgia and Germany. When comparing the WGS data from Germany with the public dataset, we found that 11 clusters contained at least one isolate from Germany and at least one isolate from another country. We could, therefore, connect TB cases despite missing epidemiological information. Conclusion: We demonstrated the added value of using WGS raw data from public repositories to contribute to TB surveillance. Comparing the German with the public dataset, we identified potential international transmission events. Thus, using this approach might support the interpretation of national surveillance results in an international context.

\section{Introduction}

Improving the surveillance of tuberculosis (TB) is one of the eight core activities identified by the World Health Organization (WHO) and the European Respiratory Society to achieve TB elimination, defined as less than one incident case per million [1]. Monitoring transmission is especially important for multidrug-resistant (MDR) Mycobacterium tuberculosis isolates - defined as being resistant to rifampicin and isoniazid - and for extensively drug-resistant (XDR) M. tuberculosis isolates - defined as MDR isolates with additional resistance to at least one of the fluoroquinolones and at least one of the second-line injectable drugs. In 2017, the WHO estimated that worldwide more than 450,000 people fell ill with MDR-TB and among these, more than 38,000 fell ill with XDR-TB [2].

The rapid advance in molecular typing technology especially the availability of whole genome sequencing (WGS) to identify and characterise pathogens - gives us the chance to integrate this information into disease surveillance. For TB surveillance, it is possible to combine the results of molecular typing of isolates from the $M$. tuberculosis complex with traditional epidemiological information to infer or to exclude TB transmission $[3,4]$. This is of particular relevance if transmission occurs among multiple countries, where epidemiological data such as social contacts are more difficult to get and where data exchange is more difficult to organise. The European Centre for Disease Prevention and Control (ECDC) reported 44 events of international transmission (international clusters) of MDR-TB in different European countries between 


\section{FIGURE 1}

Flowchart of the inclusion and exclusion of Mycobacterium tuberculosis isolates in our study from the public and the German dataset, $1996-2016^{\mathrm{a}}(\mathrm{n}=9,256)$

Public dataset

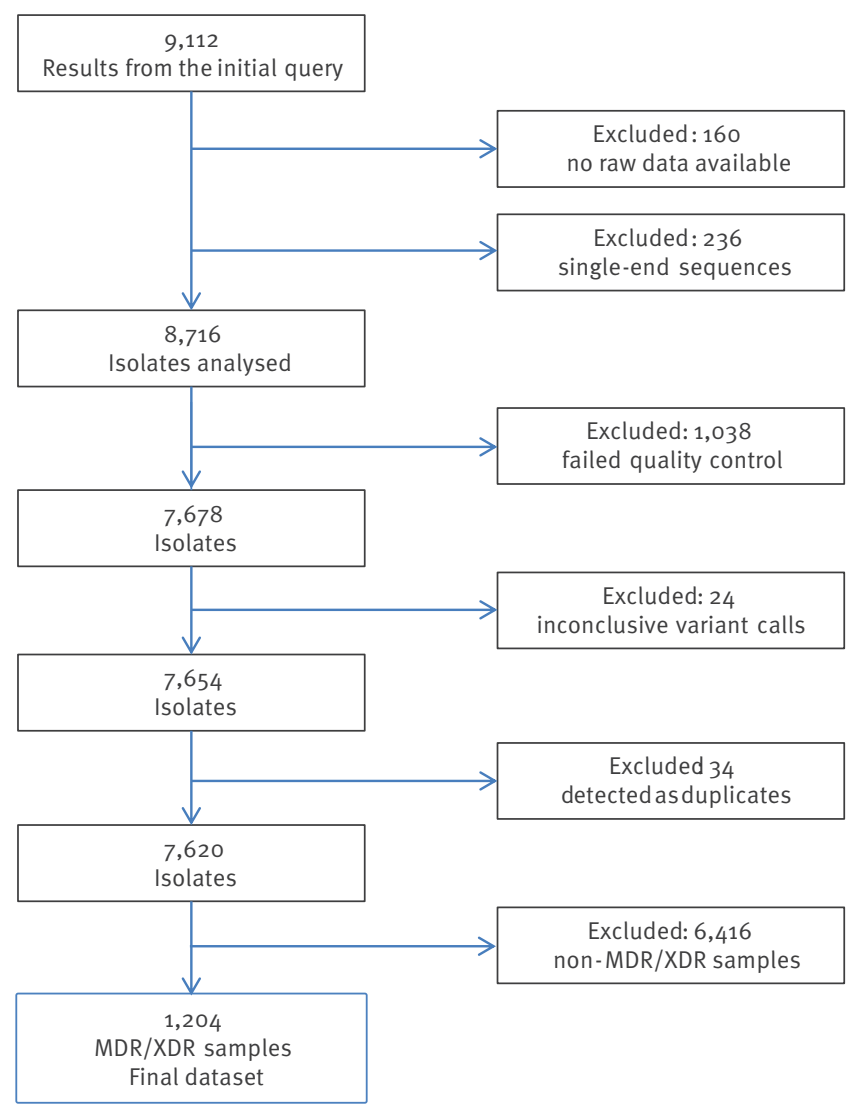

German dataset

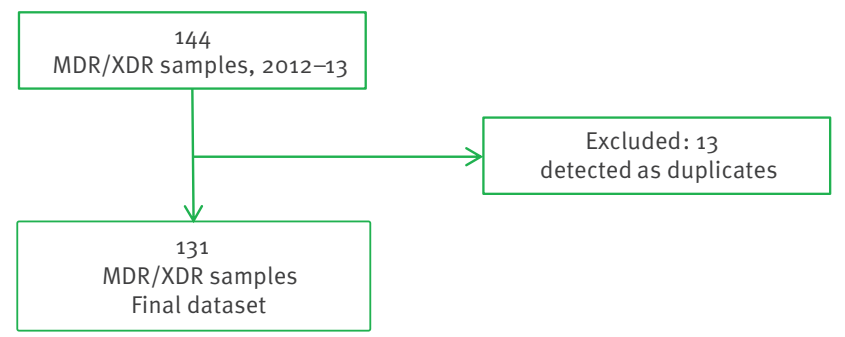

MDR: multidrug-resistant; XDR: extensively drug-resistant.

The final dataset included 1,335 isolates: 1,204 from the public and 131 from the German dataset.

a The isolates from the German dataset covered the period 2012-13.

2012 and 2015 [5]. In that report, the authors inferred TB transmission using the mycobacterial interspersed repetitive units variable number of tandem repeats (MIRU-VNTR) typing method. However, this method has limitations such as low correlation with epidemiological information in outbreak settings and low discriminatory power [3,6]. In comparison, WGS analysis offers a much higher discriminatory power and allows inferring (or excluding) TB transmission at a higher resolution [4]. In a recent systematic review, van der Werf et al. identified three studies that used WGS to investigate the international transmission of TB [7].

In recent years, the amount of available WGS data is increasing, especially because sequencing has become cheaper [8]. In addition, more and more authors deposit the raw data of their projects in open access public repositories such as the Sequence Read Archive (SRA) of the National Center for Biotechnology Information (NCBI) [9]. These publicly available raw WGS data for thousands of isolates enable the re-use and the additional analyses at a large and global scale [10]. For example, it is possible to compare genomic data among different studies or countries since the data are available in a single place. Moreover, new software tools can be tested using the same raw WGS data [11]. However, standards in bioinformatics analysis and interpretation of these WGS data for surveillance purposes are not yet fully established [12].

We aimed to assess the usefulness of raw WGS data of global MDR/XDR M. tuberculosis isolates available in public repositories to improve TB surveillance. Specifically, we wanted to identify potential international events of TB transmission and to compare the international isolates with a collection of $M$. tuberculosis isolates collected in Germany in 2012 and 2013. 
TABLE 1

Characteristics of multi- and extensively drugresistant Mycobacterium tuberculosis isolates from the public dataset analysed in this study, 1996-2016 $(\mathrm{n}=1,204)$

\begin{tabular}{|c|c|c|}
\hline \multicolumn{2}{|l|}{ Characteristic } & \multirow{2}{*}{$\begin{array}{l}\mathrm{n} \\
295 \\
\end{array}$} \\
\hline \multirow{17}{*}{ Country of isolation } & South Africa & \\
\hline & Georgia & 160 \\
\hline & Moldova & 135 \\
\hline & Vietnam & 68 \\
\hline & Azerbaijan & 57 \\
\hline & Bangladesh & 46 \\
\hline & Romania & 37 \\
\hline & Djibouti & 31 \\
\hline & Ivory Coast & 29 \\
\hline & India & 28 \\
\hline & Nigeria & 27 \\
\hline & Thailand & 24 \\
\hline & Peru & 23 \\
\hline & China & 23 \\
\hline & Tanzania & 17 \\
\hline & Other & 49 \\
\hline & NA & 155 \\
\hline \multirow{16}{*}{ Year of isolation } & 2016 & 53 \\
\hline & 2015 & 254 \\
\hline & 2014 & 106 \\
\hline & 2013 & 147 \\
\hline & 2012 & 86 \\
\hline & 2011 & 60 \\
\hline & 2010 & 87 \\
\hline & 2009 & 65 \\
\hline & 2008 & 27 \\
\hline & 2007 & 11 \\
\hline & 2006 & 6 \\
\hline & 2005 & 14 \\
\hline & 2004 & 6 \\
\hline & 2003 & 1 \\
\hline & 1996 & 1 \\
\hline & NA & 280 \\
\hline \multirow{4}{*}{ Sample type } & Sputum & 833 \\
\hline & Tissue sample from autopsy & 167 \\
\hline & Other $^{\mathrm{a}}$ & 6 \\
\hline & NA & 198 \\
\hline
\end{tabular}

NA: not available.

a E.g. bronchoalveolar lavage, lymph node.

\section{Methods}

Data collection: public dataset

The SRA database is a public repository provided by the NCBI National Library of Medicine, Bethesda, United States (US) which stores raw sequencing data derived from high-throughput sequencing platforms [9]. We queried the repository (last access: 1 March 2019) for the pathogen 'Mycobacterium tuberculosis' with samples isolated between 1996 and 2016 and restricted the results to 'genomic', 'WGS' data from the 'Illumina' sequencing technology using the appropriate query keywords. After excluding single end-sequenced and missing raw data, 8,716 isolates remained, which were further filtered for sequence characteristics. We excluded samples with reads shorter than $100 \mathrm{bp}$ as well as samples with a low $(<20 x)$ or high $(>500 x)$ average coverage depth of the reference genome (see below) to obtain a more homogenous dataset. In addition, we excluded samples with less than $90 \%$ reads aligned to the reference genome to prevent having contaminated or incorrectly annotated samples in the set. We also excluded samples for which more than $50 \%$ of all detected single-nucleotide variants were inconclusive (see Supplementary Material for details). To identify duplicates (e.g. the same file uploaded more than once in different projects) within the public dataset, we compared numbers of reads and detected variants at every step of the analysis. We excluded duplicate samples that were identical in all those numbers and their corresponding epidemiological data. After all filtering steps, 7,620 isolates remained and we will refer to these isolates as the 'public dataset' throughout the manuscript. In addition to the raw reads, we also collected metadata available in the SRA repository [9] (for details see Supplementary Table S1).

\section{Data collection: German dataset}

In addition to the international public dataset, we analysed isolates from Germany, which will be referred to as 'German dataset' throughout the manuscript. The German dataset included all $M$. tuberculosis complex isolates processed at the National Reference Center for Mycobacteria (Forschungszentrum Borstel, Germany) and classified as MDR-TB or XDR-TB in 2012 and 2013 by drug susceptibility tests (DST) according to the German TB surveillance system [13]. We chose 2012 and 2013 because for those two years, we had all corresponding epidemiological metadata, which are not available from the German TB surveillance system by default. We extracted the epidemiological data available for these isolates using the laboratory identification number of the National Reference Center for Mycobacteria. Then, we identified the respective isolate in the national surveillance system at the Robert Koch Institute (the German public health institute) and matched molecular with epidemiological data. We collected information on year of isolation, federal state of isolation, DST results, and patient-related information such as age, sex, citizenship and country of birth. 
TABLE 2

Characteristics of multidrug- and extensively drugresistant Mycobacterium tuberculosis isolates analysed in this study, Germany, 2012-2013 ( $\mathrm{n}=131)$

\begin{tabular}{|c|c|c|}
\hline \multicolumn{2}{|l|}{ Characteristic } & $\mathrm{n}$ \\
\hline \multirow{3}{*}{$\begin{array}{l}\text { Molecular drug resistance } \\
\text { prediction }\end{array}$} & MDR & 111 \\
\hline & XDR & 16 \\
\hline & Non-MDR & 4 \\
\hline \multirow{3}{*}{$\begin{array}{l}\text { Phenotypic drug resistance } \\
\text { prediction }\end{array}$} & MDR & 122 \\
\hline & XDR & 7 \\
\hline & NA & 2 \\
\hline \multirow{3}{*}{ Year of isolation } & 2013 & 80 \\
\hline & 2012 & 50 \\
\hline & 2014 & 1 \\
\hline \multirow{12}{*}{ Federal state of isolation } & $\begin{array}{c}\text { North } \\
\text { Rhine-Westphalia } \\
\end{array}$ & 32 \\
\hline & Bavaria & 13 \\
\hline & $\begin{array}{c}\text { Baden- } \\
\text { Württemberg }\end{array}$ & 15 \\
\hline & Saxony & 10 \\
\hline & Lower Saxony & 10 \\
\hline & Berlin & 10 \\
\hline & Hamburg & 8 \\
\hline & Hesse & 8 \\
\hline & $\begin{array}{l}\text { Schleswig- } \\
\text { Holstein }\end{array}$ & 5 \\
\hline & Saxony-Anhalt & 5 \\
\hline & Other states & 11 \\
\hline & NA & 4 \\
\hline \multirow{2}{*}{ Patient age (years) } & Median & $34(2-83)$ \\
\hline & Mean & 35.73 \\
\hline \multirow{3}{*}{ Patient sex } & Male & 79 \\
\hline & Female & 50 \\
\hline & NA & 2 \\
\hline \multirow{9}{*}{ Patient citizenship } & Germany & 30 \\
\hline & Russia & 25 \\
\hline & India & 8 \\
\hline & Georgia & 7 \\
\hline & Romania & 7 \\
\hline & Kazakhstan & 6 \\
\hline & Ukraine & 5 \\
\hline & Other & 39 \\
\hline & NA & 4 \\
\hline \multirow{9}{*}{ Patient country of birth } & Russia & 27 \\
\hline & Germany & 19 \\
\hline & Romania & 10 \\
\hline & Ukraine & 8 \\
\hline & India & 8 \\
\hline & Kazakhstan & 8 \\
\hline & Georgia & 7 \\
\hline & Other & 41 \\
\hline & NA & 3 \\
\hline
\end{tabular}

MDR: multidrug-resistant; XDR: extensively drug-resistant; NA: not available.

We found demographic information, epidemiological information and drug susceptibility test results in the German tuberculosis surveillance system for 129 of 131 isolates.
Whole genome sequencing analysis workflow Raw reads were subjected to quality control with Trimmomatic [14] and Flash [15]. The trimmed and filtered reads were mapped to two different reference genomes: the $M$. tuberculosis $\mathrm{H}_{37} \mathrm{Rv}$ strain and a pan-genome reference built from $146 \mathrm{M}$. tuberculosis genomes $[16,17]$ with Burrow-Wheeler algorithm bwa-mem [18]. Duplicated reads were marked and reads with mapping quality less than 10 were excluded. The Genome Analysis Toolkit (GATK) [19] was used to detect variants mapped to both reference genomes and to extract all SNPs of high quality (see Supplementary Material for details).

\section{Drug-resistance prediction}

We used Phyresse [20] and TBDreamDB [21] to identify drug resistance mutations in our datasets (last access: 18 October 2018). We filtered both lists to include only single nucleotide substitutions. For TBDreamDB, we mapped the provided locations within resistance genes to positions on the M. tuberculosis $\mathrm{H}_{37} \mathrm{Rv}$ genome where necessary. We excluded mutations not associated with drug resistance according to the WHO [22] and to the CRyPTIC study [23] (see Supplementary Table S2 for the list of all identified mutations and, among those, all the excluded mutations). For each sample, we intersected the variants detected by read mapping to the $M$. tuberculosis $\mathrm{H}_{37} \mathrm{Rv}$ genome with this list of known mutations to identify resistanceassociated mutations. We also identified uncovered or low-quality regions that overlapped with locations of resistance mutations. For the classification of isolates into resistance classes (MDR-TB and XDR-TB), we used the definitions of the WHO [2].

\section{Molecular clustering}

We used PANPASCO [17] to calculate relative pairwise single nucleotide polymorphism (SNP) distance between all isolates classified as MDR-TB or XDR-TB in the public and German dataset. This method builds on two parts to enable distance calculation for large, diverse datasets: mapping all reads to a computational pan-genome including $146 \mathrm{M}$. tuberculosis genomes and distance calculation for each individual pair of samples. For this, we identified all positions with high quality for each pair of samples and calculated the SNP distance based on this set of positions (for details on the filtering workflow, PANPASCO and distance calculation see Supplementary Material). SNPs in repeat-rich genes were not used for distance calculations as studies have shown that variants found in these regions are often false positives $[3,24]$. The list of genes provided by Comas et al. was used for filtering [25].

We applied single-linkage agglomerative clustering for defining transmission clusters and used a threshold of fewer than 13 SNPs, based on a previous study [26]. We chose the threshold of 13 SNPs (or $\geq 13$ SNPs) as cluster exclusion criteria because we aimed to identify larger events of international transmission of TB, in contrast to a threshold of 6 SNPs (or $\geq 6$ SNPs), which 


\section{FIGURE 2}

Visualisation of the transmission Cluster 2 identified among the Mycobacterium tuberculosis isolates analysed in our study, $1996-2016(n=56)$

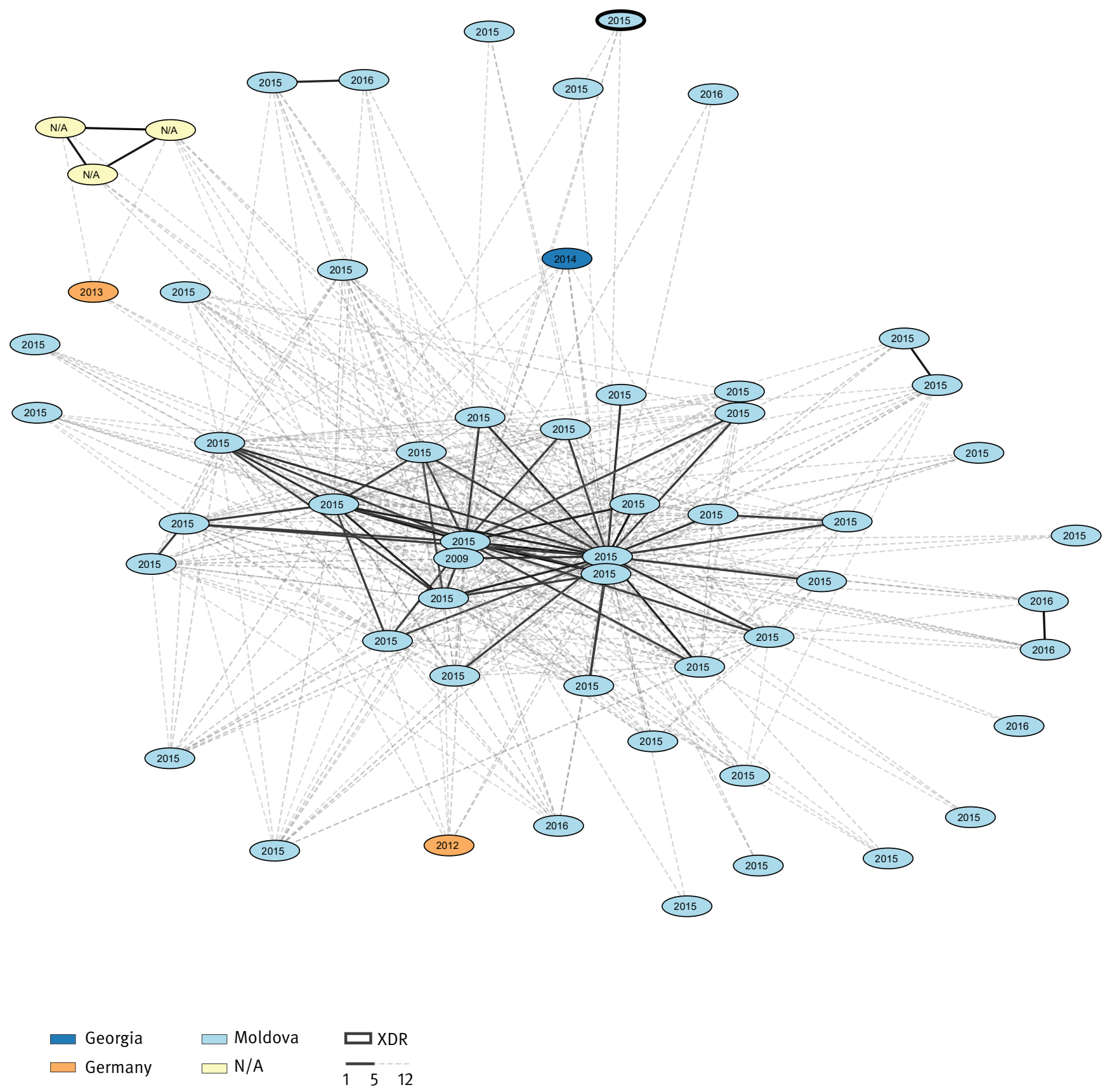

The country of isolation, multi- and extensive drug-resistance classification and year of isolation are represented in the clusters. SNP distances were calculated for each pair of isolates individually. Links with fewer than 6 SNPs are marked black, those with fewer than 13 SNPS are marked in grey. Connections with 13 SNPs or more than are not shown in the network.

might be more useful to identify recent transmission of TB $[27,28]$. Besides, we chose the threshold of 13 SNPS because our isolates were spread in terms of location and time (see below) and because we were probably missing several intermediary isolates (and cases) in our collection. PANPASCO calculates distances based on data available for each pair separately. For this reason, an individual sample can potentially have small distances to samples that have a much greater distance in direct comparison, owing to a higher number of compared high-quality sites. In this study, we aimed to discover clusters of closely related samples. Therefore, the implemented agglomerative clustering approach evaluated the distance from the sample that should be added to two instead of one sample of an existing cluster - we compared not only pairs of samples but two sets of trios. The sample was added to the cluster only if the maximum distance in the trio was below twice the SNP threshold. Samples that violated this condition 
Characteristics of the 11 molecular clusters identified in this study which contain at least one isolate from Germany and at least one isolate from another country, $1996-2016^{\mathrm{a}}(\mathrm{n}=140)$

\begin{tabular}{|c|c|c|c|c|c|c|c|}
\hline \multirow{2}{*}{$\begin{array}{l}\text { Cluster } \\
\text { name }\end{array}$} & \multirow{2}{*}{$\begin{array}{l}\text { Number of } \\
\text { isolates in the } \\
\text { cluster }\end{array}$} & \multirow{2}{*}{$\begin{array}{l}\text { Number of } \\
\text { MDR isolates }\end{array}$} & \multirow{2}{*}{$\begin{array}{l}\text { Country of isolation of } \\
\text { MDR (n) }\end{array}$} & \multirow{2}{*}{$\begin{array}{c}\text { Number of } \\
\text { XDR isolates }\end{array}$} & \multirow{2}{*}{$\begin{array}{l}\text { Country of isolation } \\
\text { of XDR (n) }\end{array}$} & \multicolumn{2}{|c|}{$\begin{array}{l}\text { Characteristics of isolates from the } \\
\text { German dataset within the clusters }\end{array}$} \\
\hline & & & & & & $\begin{array}{l}\text { Patient country } \\
\text { of birth (n) }\end{array}$ & $\begin{array}{c}\text { Patient } \\
\text { nationality (n) }\end{array}$ \\
\hline 2 & 56 & 55 & $\begin{array}{c}\text { Moldova (49) } \\
\text { Germany (2) } \\
\text { Georgia (1) } \\
\text { NA (3) }\end{array}$ & 1 & Moldova (1) & $\begin{array}{l}\text { Romania (1) } \\
\text { Germany (1) }\end{array}$ & $\begin{array}{l}\text { Romania (1) } \\
\text { Germany (1) }\end{array}$ \\
\hline 5 & 30 & 12 & $\begin{array}{c}\text { South Africa (11) } \\
\text { Germany (1) }\end{array}$ & 18 & South Africa (18) & Abroad (1) & Abroad (1) \\
\hline 9 & 18 & 18 & $\begin{array}{l}\text { Georgia (16) } \\
\text { Germany (2) }\end{array}$ & 0 & 0 & $\begin{array}{l}\text { Georgia (1) } \\
\text { Romania (1) }\end{array}$ & $\begin{array}{l}\text { Georgia (1) } \\
\text { Germany (1) }\end{array}$ \\
\hline 13 & 10 & 1 & Germany (1) & 9 & Kazakhstan (9) & Kazakhstan (1) & Germany (1) \\
\hline 21 & 6 & 6 & $\begin{array}{l}\text { Georgia (5) } \\
\text { Germany (1) }\end{array}$ & 0 & 0 & Syria (1) & Syria (1) \\
\hline 24 & 5 & 5 & $\begin{array}{l}\text { Georgia (3) } \\
\text { Germany (2) }\end{array}$ & 0 & 0 & Georgia (2) & Georgia (2) \\
\hline 35 & 4 & 1 & Georgia (1) & 3 & $\begin{array}{l}\text { Georgia (2) } \\
\text { Germany (1) }\end{array}$ & Georgia (1) & Georgia (1) \\
\hline 53 & 3 & 2 & $\begin{array}{l}\text { Romania (1) } \\
\text { Germany (1) }\end{array}$ & 1 & Romania (1) & Romania (1) & Romania (1) \\
\hline 58 & 3 & 3 & $\begin{array}{c}\text { India (2) } \\
\text { Germany (1) }\end{array}$ & 0 & 0 & India (1) & India (1) \\
\hline 59 & 3 & 3 & $\begin{array}{l}\text { Georgia (1) } \\
\text { Germany (2) }\end{array}$ & o & 0 & $\begin{array}{l}\text { Georgia (1) } \\
\text { Ukraine(1) }\end{array}$ & $\begin{array}{l}\text { Georgia (1) } \\
\text { Ukraine(1) }\end{array}$ \\
\hline 103 & 2 & 2 & $\begin{array}{l}\text { Georgia (1) } \\
\text { Germany (1) }\end{array}$ & 0 & 0 & Georgia (1) & Georgia (1) \\
\hline
\end{tabular}

MDR: multidrug-resistant; XDR: extensively drug-resistant; NA: not available.

In bold the isolates from Germany. Within each cluster, information about the country of birth and nationality of the patient are provided.

a The isolates from the German dataset covered the period 2012-13.

were iteratively removed from the clustering and were marked for potential follow-up analyses.

We used Cytoscape 3.7 to visualise the clusters [29]. We classified all clustered samples into TB lineages using lineage-specific SNPs provided in [30] and [31] (see Supplementary Table S6). We compared and validated clustering results of a subset of isolates using the pipeline MTBSeq [32] (see Supplementary Table S7).

\section{Data availability}

The raw WGS data used in this study are available in the NCBI SRA repository. The accession numbers for all samples of the public dataset are available in Supplementary Table S1. The German dataset is available as Bioproject PRJEB35201. Software for creating a pan-genome sequence (seq-seq-pan) is accessible at https://gitlab.com/rki_bioinformatics/seq-seq-pan, and scripts for the NGS workflow and the SNP-distance method (PANPASCO) are available at https://gitlab. com/rki_bioinformatics/panpasco. The code for the clustering method is available at https://gitlab.com/ rki_bioinformatics/snp_distance_clustering.

\section{Ethical statement}

Ethical approval was not required for this study since data were extracted from pseudonymised notification data. 


\section{Results}

\section{Final dataset}

After the filtering steps, 7,620 of initially 8,716 downloaded isolates remained in the public dataset and 131 isolates in the German dataset (Figure 1). We focused our study on MDR/XDR-TB, and therefore the final dataset contained overall 1,335 isolates after filtering using resistance-associated SNPS. Supplementary Table $\mathrm{S} 1$ shows the cluster assignment, molecular drug resistance prediction and extracted metadata of these 1,335 isolates.

\section{Metadata availability and drug-resistance} prediction: public dataset $(n=1,204)$

The majority of metadata collected from the public dataset consisted of the country of isolation $(1,049 / 1,204$; $87.13 \%)$, the year of isolation (921/1,204; 76.50\%) and the sample type $(997 / 1,204 ; 82.81 \%)$ (Table 1). For other metadata, we could collect less information, for example for patient age (174/1,204; 14.45\%), patient sex $(171 / 1,204 ; 14.20 \%)$, or patient HIV status (157/1,204; 13.04\%) (Supplementary Table S1). For 912 isolates, we had information on both country and year of isolation. Initially, we identified 336 isolates with missing data for the country of isolation. After examining the Bioproject information (SRA [9]) for these 336 isolates, we could identify the country of isolation for a further 177 isolates, leaving us with 155 isolates without any information regarding the country of isolation. We identified 970 MDR (80.56\%) and 234 XDR (19.44\%) isolates.

\section{Metadata availability and drug resistance} prediction: German dataset $(\mathrm{n}=131)$

We could retrieve demographics, epidemiological information and DST results for 129 of 131 (98.47\%) of the isolates from the German TB surveillance system. Characteristics of the molecular clusters for the German data set are shown in Table 2. Supplementary Table S3 shows the corresponding collected metadata for the public data set. The 131 German isolates came from 15 of the 16 German federal states. The most frequent countries of birth of the patients were Russia (27/131; 20.61\%), Germany (19/131; 14.50\%) and Romania (10/131; 7.63\%) (Table 2$)$.

We noted discrepancies in the identification of rifampicin resistance between the results of the phenotypic DST and the detection of drug resistance mutations in 13 isolates (documented in Supplementary Table S3). Specifically, four isolates were classified as MDR in the TB surveillance system while they were classified as non-MDR according to the molecular analysis because they did not contain any known drug resistance mutations against rifampicin. However, in one of these four isolates we found insufficient sequencing coverage in some of the genomic regions with known resistance mutations for rifampicin; while in another isolate we found an insertion of $3 \mathrm{nt}$ near a region with known resistance mutations for rifampicin.
In addition, nine isolates were classified as MDR in the TB surveillance system, while they were classified as XDR according to the analysis of the drug resistance mutations. The reason for such discrepancy was that a drug resistance mutation against amikacin, kanamycin or capreomycin was identified in these 10 isolates, but no DST results were available for these antibiotics.

\section{Molecular clustering and comparison between the public and the German dataset}

Among all isolates of our study, we identified 133 molecular clusters (with at least two isolates) and 591 singletons. The 133 clusters included 744 isolates (Supplementary Table S4). Supplementary Table S5 shows a summary of distances between all isolates for all molecular clusters. In 16 clusters, the isolates were from at least two different countries of isolation, suggesting larger events of international transmission of TB (Supplementary Table S4). For example, Cluster 2 included $56 \mathrm{MDR} / \mathrm{XDR}$ isolates collected in three countries - Georgia $(n=1)$, Germany $(n=2)$ and Moldova $(n=50)$, including three samples without information on country of collection. A total of 51 of the 56 isolates in this cluster were part of a previous study (Bioproject PRJNA318002 [33], Supplementary Table S1). In Figure 2 we show the country of isolation and the year of isolation of the isolates belonging to Cluster 2 .

Cluster 1 was the largest cluster $(n=79)$ identified in our study. According to the metadata such as host subject (the patient identification number), isolate name, year of isolation, patient age, and patient sex (see Supplementary Table $\mathrm{S}_{1}$ ), the isolates were 79 autopsy samples from different anatomic sites (such as lung or liver) of the same patient, marked as P21. Similarly, Cluster 3, Cluster 14, Cluster 16, Cluster 18 and Cluster 28 contained multiple isolates from single patients from South Africa, which were part of a study including 2,693 autopsy samples of 44 subjects [34]. In line with previous findings [34], our analysis showed very low variability within the South African clusters, highlighted by the low maximum cluster distances in each of these 'single-patient' clusters (Supplementary Table S5). Also, Cluster 26, Cluster 32 and Cluster 33 included multiple isolates from single patients. These isolates were part of a study investigating the evolution of drug-resistant TB in patients during long-term treatment [35].

When we compared the German dataset with the public dataset, we observed that in 11 clusters, there was at least one isolate from Germany and at least one isolate from another country. Table 3 shows the relation between the German isolates and the international isolates from the public dataset. The epidemiological information collected from the German isolates correlates well with molecular clusters in seven of 11 cases. For example in Cluster 9, there were 16 isolates from Georgia and two isolates from Germany; the country of birth recorded for one of the two patients from Germany was Georgia. Also Cluster 24, Cluster 35 
and Cluster 103 included isolates from Georgia and Germany, and the country of birth recorded for the patients from Germany was Georgia. Three further examples of agreement between molecular and epidemiological data were: Cluster 13 which included isolates from Germany and Kazakhstan, Cluster 53 which included isolates from Germany and from Romania, and Cluster 58 which included isolates from Germany and India (Table 3). By comparing the molecular data of the German and the public dataset, we could connect cases that had previously not been epidemiologically linked. For example in Cluster 2 (Figure 2), two isolates from Germany (in orange) were connected through several isolates from Georgia and Moldova (in dark and light blue), and the distance between the two German isolates was $\geq 13$ SNPs. Similarly, in the Cluster 53, two isolates from Romania were connected through a German isolate, and the distance between the two isolates from Romania was $\geq 13$ SNPs (data not shown).

\section{Discussion}

In this study, we assessed whether raw WGS data of MDR-/XDR M. tuberculosis isolates available from public sequence repositories are useful to improve TB surveillance. We identified several molecular clusters including isolates from multiple countries, suggesting larger events of international transmission of TB. We expected to find international TB transmission events, also considering previous studies reporting crossborder molecular clusters $[5,7]$. Looking at the collected metadata, we identified several clusters with multiple isolates from the same patient or multiple autopsy samples collected from the same patient [34,35]. This shows the importance of providing complete metadata together with the publicly available molecular data. Based on the metadata, we could distinguish between clusters of isolates taken from different patients - the real transmission clusters - and clusters of isolates taken from a single patient. The real transmission clusters are crucial for the routine TB surveillance, while the clusters of isolates taken from the same patient are useful to study the intra-host variability of isolates.

We observed agreement between molecular and epidemiological data by comparing the public and the German datasets. This is clear for example in the clusters originating from Georgia, which contained isolates from both the German dataset and the public dataset. It is therefore likely that migrants from Georgia acquired the TB infections in their home country - or during visits there - and were diagnosed later when they moved or returned to Germany, as already described [36]. This shows that we could identify events of potential international transmission (between Germany and Georgia) that we could have missed by looking only at the German molecular clusters. Our analysis can have implications for surveillance and public health, for example by linking TB patients from different countries during contact tracing procedures. In the best scenario, where we could compare the German and the public dataset in (almost) real time, we would detect international transmission of TB earlier and inform the public health authorities timely.

We observed discrepancies in the identification of rifampicin resistance between the phenotypic DST and the detected drug resistance mutations. Specifically, four isolates were phenotypically resistant to rifampicin but they did not contain any known drug resistance mutations against rifampicin or the genetic regions containing the known mutation had lower sequencing quality. This means that in our study, the known drug resistance mutations (there might be always new mutations conferring resistance) correctly predicted the resistance to rifampicin in 125 of 129 isolates, resulting in a sensitivity of $96.9 \%$. This sensitivity is in accordance with a study by the CRYPTIC Consortium, where the authors reported a sensitivity of $97.5 \%$ [23]. Misclassification occurred in four isolates, which were MDR by phenotype, but non-MDR by genotype. This might have had consequences for patient therapy if we had replaced the phenotypic DST with the molecular detection of drug resistance mutations. Therefore, we suggest being careful in the transition from phenotypic to genotypic drug resistance determination as suggested by the CRYPTIC Consortium [23]. Specifically, laboratories and national reference laboratories should still perform the phenotypic DST, for example on a representative set of isolates or isolates with low sequencing quality and coverage.

Our study has three major limitations: firstly, the raw WGS data uploaded in the SRA repository [9] were either from single studies or from outbreaks and therefore, they were not representative of the TB situation in the different countries. Besides, we are probably missing several intermediary isolates (and cases) in our collection. These examples of sampling biases are, however, well-known biases in molecular epidemiology studies [37]. Secondly, the collected metadata were incomplete, especially regarding patient information. Both limitations can be overcome by genotyping all TB isolates, by including the genotyping results in the TB surveillance systems and by making genotyping data publicly available. Thirdly, we relied on single-linkage clustering, as this is currently a widely used approach for transmission cluster detection [38]. However, with many missing samples (which is probably the case when using a public repository), single-linkage can become unreliable and results strongly dependent on the cut-off and sample coverage for the specific cluster. For our study and its exploratory purpose, we preferred to use a widely accepted cluster detection approach combined with a higher cut-off. Therefore, we preferred to err on the side of caution (identifying a false positive connection) rather than miss a potential transmission. Thus, in future studies, researchers should carefully evaluate the clustering methods for transmission cluster detection with missing data. 


\section{Conclusion}

Our study has one major implication: we demonstrated that by considering the international context (the public dataset), while analysing the national molecular data (the German dataset), we could identify previously unknown transmissions between patients. Thus, we could detect larger and international events of TB transmission. To improve the WGS-based TB surveillance we therefore suggest to regularly compare the national molecular clusters with the international molecular clusters available in the public sequence repositories. Lastly, supranational institutions such as the WHO, the ECDC or international TB networks could perform such analyses at a global scale, improving the global surveillance of TB.

\section{Acknowledgements}

We would like to thank Birgit Voß for her help in matching epidemiological and molecular data. We want to thank the National Reference Center for Mycobacteria in Borstel, Germany for acquiring the sequencing data for the German dataset. We thank Lena Fiebig and Marta Andrés for their initial input in the study.

\section{Conflict of interest}

None declared.

\section{Authors' contributions}

Andrea Sanchini: participated in the study design, participated in the data collection, analysed the data, interpreted the results and wrote the manuscript. Christine Jandrasits: designed the study, collected the data, analysed the data, interpreted the results and wrote the manuscript. Julius Tembrockhaus: collected the data, analysed the data and revised the manuscript. Thomas Andreas Kohl: participated in data analysis, and interpretation of the results, and revised the manuscript. Christian Utpatel: participated in data analysis, interpretation of the results, and revised the manuscript. Florian P. Maurer: participated in data analysis, and revised the manuscript. Stefan Niemann: participated in study design and revised the manuscript. Walter Haas: designed the study, participated in the interpretation of the results and revised the manuscript. Bernhard Y. Renard: designed the study, participated in the interpretation of the results, coordinated the project and revised the manuscript. Stefan Kröger: designed the study, participated in the data analysis, participated in the interpretation of the results, coordinated the project and revised the manuscript.

\section{References}

1. Matteelli A, Rendon A, Tiberi S, Al-Abri S, Voniatis C, Carvalho ACC, et al. Tuberculosis elimination: where are we now? Eur Respir Rev. 2018;27(148):180035. https://doi. org/10.1183/16000617.0035-2018 PMID: 29898905

2. World Health Organization (WHO). Global tuberculosis report 2019. Geneva: WHO; 2019. Available from: https://apps.who. int/iris/bitstream/handle/10665/329368/9789241565714-eng. pdf?ua $=1$

3. Roetzer A, Diel R, Kohl TA, Rückert C, Nübel U, Blom J, et al. Whole genome sequencing versus traditional genotyping for investigation of a Mycobacterium tuberculosis outbreak: a longitudinal molecular epidemiological study. PLoS Med. 2013;10(2):e1001387. https://doi.org/10.1371/journal. pmed.1001387 PMID: 23424287
4. Hatherell H-A, Colijn C, Stagg HR, Jackson C, Winter JR, Abubakar I. Interpreting whole genome sequencing for investigating tuberculosis transmission: a systematic review. BMC Med. 2016;14(1):21. https://doi.org/10.1186/s12916-016 0566-x PMID: 27005433

5. European Centre for Disease Prevention and Control (ECDC). Molecular typing for surveillance of multidrug-resistant tuberculosis in the EU/EEA - March 2017. Stockholm: ECDC; 2017. Available from: https://www.ecdc.europa.eu/ sites/default/files/documents/MDR-TB-molecular-typingsurveillance-mar-2017_1.pdf

6. Wyllie DH, Davidson JA, Grace Smith E, Rathod P, Crook DW, Peto TEA, et al. A quantitative evaluation of MIRU-VNTR typing against whole-genome sequencing for identifying Mycobacterium tuberculosis transmission: a prospective observational cohort study. EBioMedicine. 2018;34:122-30. https://doi.org/10.1016/j.ebiom.2018.07.019 PMID: 30077721

7. van der Werf MJ, Ködmön C. Whole-genome sequencing as tool for investigating international tuberculosis outbreaks: a systematic review. Front Public Health. 2019;7:87. https://doi. org/10.3389/fpubh.2019.00087 PMID: 31058125

8. Muir P, Li S, Lou S, Wang D, Spakowicz DJ, Salichos L, et al. The real cost of sequencing: scaling computation to keep pace with data generation. Genome Biol. 2016;17(1):53. https://doi. org/10.1186/s13059-016-0917-0 PMID: 27009100

9. Leinonen R, Sugawara H, Shumway M, International Nucleotide Sequence Database Collaboration. The sequence read archive. Nucleic Acids Res. 2011;39(Database issue):D19-21. https:// doi.org/10.1093/nar/gkq1019 PMID: 21062823

10. Ohta T, Nakazato T, Bono H. Calculating the quality of public high-throughput sequencing data to obtain a suitable subset for reanalysis from the Sequence Read Archive. Gigascience. 2017;6(6):1-8. https://doi.org/10.1093/gigascience/gixo29 PMID: 28449062

11. Ball CA, Sherlock G, Brazma A. Funding high-throughput data sharing. Nat Biotechnol. 2004;22(9):1179-83. https://doi. org/10.1038/nbto904-1179 PMID: 15340487

12. Meehan CJ, Goig GA, Kohl TA, Verboven L, Dippenaar A, Ezewudo M, et al. Whole genome sequencing of Mycobacterium tuberculosis: current standards and open issues. Nat Rev Microbiol. 2019;17(9):533-45. "https://www.ncbi.nlm.nih. gov/entrez/query.fcgi?cmd=Retrieve $\& d b=$ PubMed\&lis t_uids=31209399\&dopt=Abstract" https://doi.org/10.1038/ S41579-019-0214-5 PMID: 31209399

13. Robert Koch Institute (RKI). RKI-Bericht zur Epidemiologie der Tuberkulose in Deutschland für 2018. [RKI report on the epidemiology of tuberculosis in Germany for 2018]. Berlin: RKI; 2019. German. Available from: https://www.rki. de/DE/Content/InfAZ/T/Tuberkulose/Download/TB2018. pdf; jsessionid $=5$ BCAC 5554 CB 57 C 583 B6 $4705148 \mathrm{FFC}$ 1Bo.1_cid372?__blob=publicationFile

14. Bolger AM, Lohse M, Usadel B. Trimmomatic: a flexible trimmer for Illumina sequence data. Bioinformatics. 2014;30(15):211420. https://doi.org/10.1093/bioinformatics/btu170 PMID: 24695404

15. Magoč T, Salzberg SL. FLASH: fast length adjustment of short reads to improve genome assemblies. Bioinformatics. 2011;27(21):2957-63. https://doi.org/10.1093/bioinformatics/ btr507 PMID: 21903629

16. Jandrasits C, Dabrowski PW, Fuchs S, Renard BY. seq-seq-pan: building a computational pan-genome data structure on whole genome alignment. BMC Genomics. 2018;19(1):47. https://doi. org/10.1186/s12864-017-4401-3 PMID: 29334898

17. Jandrasits C, Kröger S, Haas W, Renard BY. Computational pangenome mapping and pairwise SNP-distance improve detection of Mycobacterium tuberculosis transmission clusters. PLOS Comput Biol. 2019;15(12):e1007527. https://doi.org/10.1371/ journal.pcbi.1007527 PMID: 31815935

18. Li H. Aligning sequence reads, clone sequences and assembly contigs with BWA-MEM. arXiv:1303.3997 [q-bio.GN]. 2013. Available from: https://arxiv.org/abs/1303.3997

19. DePristo MA, Banks E, Poplin R, Garimella KV, Maguire JR, Hartl C, et al. A framework for variation discovery and genotyping using next-generation DNA sequencing data. Nat Genet. 2011;43(5):491-8. https://doi.org/10.1038/ng.806 PMID: 21478889

20. Feuerriegel S, Schleusener V, Beckert P, Kohl TA, Miotto P, Cirillo DM, et al. PhyResSE: a web tool delineating Mycobacterium tuberculosis antibiotic resistance and lineage from whole-genome sequencing data. J Clin Microbiol. 2015;53(6):1908-14. https://doi.org/10.1128/JCM.00025-15 PMID: 25854485

21. Sandgren A, Strong M, Muthukrishnan P, Weiner BK, Church GM, Murray MB. Tuberculosis drug resistance mutation database. PLoS Med. 2009;6(2):e2. https://doi.org/10.1371/ journal.pmed.1000002 PMID: 19209951 
22. Miotto P, Tessema B, Tagliani E, Chindelevitch L, Starks AM, Emerson C, et al. A standardised method for interpreting the association between mutations and phenotypic drug resistance in Mycobacterium tuberculosis. Eur Respir J. 2017;50(6):1701354. https://doi.org/10.1183/13993003.013542017 PMID: 29284687

23. CRYPTIC Consortium and the 100,000 Genomes ProjectAllixBéguec C, Arandjelovic I, Bi L, Beckert P, Bonnet M, et al. Prediction of susceptibility to first-line tuberculosis drugs by DNA sequencing. N Engl J Med. 2018;379(15):1403-15. https:// doi.org/10.1056/NEJMoa1800474 PMID: 30280646

24. Roetzer A, Schuback S, Diel R, Gasau F, Ubben T, di Nauta A, et al. Evaluation of Mycobacterium tuberculosis typing methods in a 4-year study in Schleswig-Holstein, Northern Germany. J Clin Microbiol. 2011;49(12):4173-8. https://doi.org/10.1128/ JCM.05293-11 PMID: 21998434

25. Comas I, Chakravartti J, Small PM, Galagan J, Niemann S, Kremer K, et al. Human T cell epitopes of Mycobacterium tuberculosis are evolutionarily hyperconserved. Nat Genet. 2010;42(6):498-503. https://doi.org/10.1038/ng.590 PMID: 20495566

26. Walker TM, Ip CLC, Harrell RH, Evans JT, Kapatai G, Dedicoat MJ, et al. Whole-genome sequencing to delineate Mycobacterium tuberculosis outbreaks: a retrospective observational study. Lancet Infect Dis. 2013;13(2):137-46. https://doi.org/10.1016/ S1473-3099(12)70277-3 PMID: 23158499

27. Kohl TA, Harmsen D, Rothgänger J, Walker T, Diel R, Niemann S. Harmonized genome wide typing of tubercle bacilli using a web-based gene-by-gene nomenclature system. EBioMedicine. 2018;34:131-8. https://doi.org/10.1016/j.ebiom.2018.07.030 PMID: 30115606

28. Jajou R, Kohl TA, Walker T, Norman A, Cirillo DM, Tagliani E, et al. Towards standardisation: comparison of five whole genome sequencing (WGS) analysis pipelines for detection of epidemiologically linked tuberculosis cases. Euro Surveill. 2019;24(50):1900130. https://doi.org/10.2807/1560-7917. ES.2019.24.50.1900130 PMID: 31847944

29. Shannon P, Markiel A, Ozier O, Baliga NS, Wang JT, Ramage D, et al. Cytoscape: a software environment for integrated models of biomolecular interaction networks. Genome Res. 2003;13(11):2498-504. https://doi.org/10.1101/gr.1239303 PMID: 14597658

30. Coll F, McNerney R, Guerra-Assunção JA, Glynn JR, Perdigão J, Viveiros $M$, et al. A robust SNP barcode for typing Mycobacterium tuberculosis complex strains. Nat Commun. 2014;5(1):4812. https://doi.org/10.1038/ncomms5812 PMID: 25176035

31. Merker M, Blin C, Mona S, Duforet-Frebourg N, Lecher S, Willery $\mathrm{E}$, et al. Evolutionary history and global spread of the Mycobacterium tuberculosis Beijing lineage. Nat Genet. 2015;47(3):242-9. https://doi.org/10.1038/ng.3195 PMID: 25599400

32. Kohl TA, Utpatel C, Schleusener V, De Filippo MR, Beckert P, Cirillo DM, et al. MTBseq: a comprehensive pipeline for whole genome sequence analysis of Mycobacterium tuberculosis complex isolates. Peerl. 2018;6:e5895. https://doi. org/10.7717/peerj.5895 PMID: 30479891

33. Rosenthal A, Gabrielian A, Engle E, Hurt DE, Alexandru S, Crudu V, et al. The TB portals: an open-access, web-based platform for global drug-resistant-tuberculosis data sharing and analysis. J Clin Microbiol. 2017;55(11):3267-82. https://doi. org/10.1128/JCM.01013-17 PMID: 28904183

34. Lieberman TD, Wilson D, Misra R, Xiong LL, Moodley P, Cohen $\mathrm{T}$, et al. Genomic diversity in autopsy samples reveals within-host dissemination of HIV-associated Mycobacterium tuberculosis. Nat Med. 2016;22(12):1470-4. https://doi. org/10.1038/nm.4205 PMID: 27798613

35. Xu Y, Liu F, Chen S, Wu J, Hu Y, Zhu B, et al. In vivo evolution of drug-resistant Mycobacterium tuberculosis in patients during long-term treatment. BMC Genomics. 2018;19(1):640. https:// doi.org/10.1186/s12864-018-5010-5 PMID: 30157763

36. Odone A, Tillmann T, Sandgren A, Williams G, Rechel B, Ingleby $D$, et al. Tuberculosis among migrant populations in the European Union and the European Economic Area. Eur J Public Health. 2015;25(3):506-12. https://doi.org/10.1093/eurpub/ cku208 PMID: 25500265

37. Murray M, Alland D. Methodological problems in the molecular epidemiology of tuberculosis. Am J Epidemiol. 2002;155(6):56571. https://doi.org/10.1093/aje/155.6.565 PMID: 11882530

38. Rodriguez MZ, Comin CH, Casanova D, Bruno OM, Amancio DR, Costa LDF, et al. Clustering algorithms: A comparative approach. PLoS One. 2019;14(1):e0210236. https://doi. org/10.1371/journal.pone.0210236 PMID: 30645617

\section{License, supplementary material and copyright}

This is an open-access article distributed under the terms of the Creative Commons Attribution (CC BY 4.0) Licence. You may share and adapt the material, but must give appropriate credit to the source, provide a link to the licence and indicate if changes were made.

Any supplementary material referenced in the article can be found in the online version.

This article is copyright of the authors or their affiliated institutions, 2021. 